\title{
ANÁLISIS
}

\section{FAKE NEWS, ¿AMENAZA U OPORTUNIDAD PARA LOS PROFESIONALES DE LA INFORMACIÓN Y LA DOCUMENTACIÓN?}

\author{
Fake news, threat or opportunity for information \\ professionals?
}

Alexandre López-Borrull, Josep Vives-Gràcia y Joan-Isidre Badell

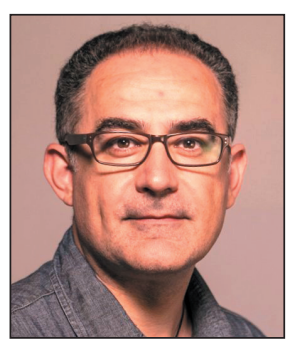

Alexandre López-Borrull es profesor agregado de la Universitat Oberta de Catalunya (UOC), donde es director de los Estudios de Información y Documentación. Es doctor en Química por la Universitat Autònoma de Barcelona y profesor ayudante en la misma universidad en el período 1998-2009. Licenciado en Documentación por la UOC. Como investigador ha trabajado en aspectos legales de la información, fuentes de información electrónica en ciencia y tecnología, y ha participado en diversos proyectos. Sus intereses de investigación están relacionados con open science, big science y e-research. Forma parte del grupo de investigación Knowledge and Information Management in Organizations (KIMO).

https://orcid.org/0000-0003-1609-2088

Universitat Oberta de Catalunya Rambla del Poble Nou, 156. 08018 Barcelona, España alopezbo@uoc.edu

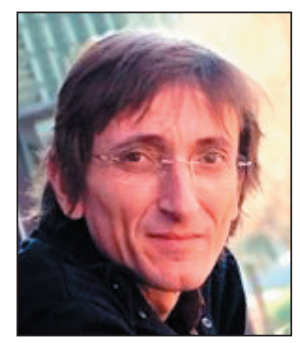

Josep Vives-Gràcia es jefe del Servei de Biblioteques del Departament de Cultura de la Generalitat de Catalunya. Licenciado en Documentación y diplomado en Biblioteconomía y Documentación, ha trabajado también en bibliotecas especializadas y universitarias. Profesor colaborador de los Estudios de Información y Documentación de la Universitat Oberta de Catalunya (UOC). Ha publicado artículos sobre ética profesional, propiedad intelectual, alfabetización informacional, y gestión bibliotecaria.

https://orcid.org/0000-0002-4132-7849

Generalitat de Catalunya, Servei de Biblioteques Plaça de Salvador Seguí, 1-9 (Edifici Filmoteca). 08001 Barcelona, España josepvives@gencat.cat

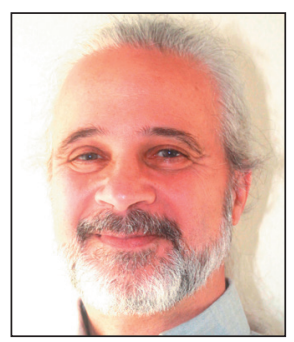

Joan-Isidre Badell, bibliotecario en la Universitat Pompeu Fabra (UPF), es profesor asociado del Departamento de Comunicación de la UPF, profesor colaborador en el Master Universitario Online de Documentación Digital, el Master Universitario de Buscadores en la Barcelona Management School (BSM-UPF) y profesor colaborador en la Universitat Oberta de Catalunya (UOC). Anteriormente fue profesor en la Universidad de Vic (UVic) (1999-2008), donde ocupó el cargo de director de departamento y de coordinador académico (2002-2005). Es diplomado en Biblioteconomía y Documentación por la Universitat de Barcelona (UB), master en Gestión de la Documentación Digital $(U B)$ y doctor en Información y Documentación en la Sociedad del Conocimiento (UB). Investigador colaborador de DigiDoc (Documentación Digital) de la UPF desde 2006.

https://orcid.org/0000-0001-8793-6722

Universitat Pompeu Fabra, Biblioteca Ramon Trias Fargas, 25-27. 08005 Barcelona, España joanisidre.badell@upf.edu

\section{Resumen}

Presentamos en este artículo una aproximación contextual del fenómeno de las noticias falsas en relación con el campo de la información y la documentación y el papel que los profesionales del sector podemos ejercer eficaz y eficientemente. 
Hacemos una descripción de iniciativas y proyectos, tanto de las instituciones bibliotecarias y de sus profesionales, como de los sectores de la educación y de la comunicación, también afectados e involucrados en la problemática de las noticias falsas y de la posverdad. En las conclusiones planteamos la necesaria revisión de una serie de prácticas y actividades desarrolladas hasta ahora, la participación y colaboración con otros sectores profesionales implicados, y la potenciación de proyectos de formación en competencias digitales y mediáticas.

\title{
Palabras clave
}

Noticias falsas; Posverdad; Ética profesional; Profesionales de la información y la documentación; Bibliotecarios-documentalistas; Alfabetización en medios; Alfabetización informacional.

\begin{abstract}
A contextual approach to the phenomenon of false news in the field of information and documentation is presented, taking into account the role that professionals in the sector can exercise efficiently. We present a description of projects, both from library institutions and their professionals, and from the education and communication sectors, also affected and involved in the problem of fake news and post-truth. In the conclusions the required revision of a series of practices and activities so far developed is stated, with both the participation and the collaboration with other professional sectors involved, and the implementation of training projects in digital and media literacy.
\end{abstract}

\section{Keywords}

Fake news; Information professionals; Professional ethics; Librarians; Media literacy; Informational literacy; Post-truth.

López-Borrull, Alexandre; Vives-Gràcia, Josep; Badell, Joan-Isidre (2018). "Fake news, ¿amenaza u oportunidad para los profesionales de la información y la documentación?". El profesional de la información, v. 27, n. 6, pp. 1346-1356.

https://doi.org/10.3145/epi.2018.nov.17

\section{Introducción}

Tradicionalmente los profesionales de la información han tenido a su alcance una serie de fuentes de información que se consideraban fiables y a las que podían recurrir para dar respuesta a las necesidades de sus usuarios. Una fuente confiable, de referencia, seguía una serie de controles y validaciones que permitían garantizar la calidad de la información que se podía encontrar. A partir de la irrupción de internet, del blog, la web, etc., el monopolio de comunicar información se rompe y ya no sólo los editores de periódicos, revistas, libros o medios de comunicación tendrán la capacidad de emitir sus mensajes (Casero-Ripollés; López-Meri, 2015).

La consolidación de las redes sociales fácilmente usables, especialmente Twitter y Facebook, ha permitido el crecimiento de las noticias falsas (fake news) que ahora mismo son un verdadero problema a la hora de determinar la certeza o no de una información. A partir de 2016, con mucho énfasis durante la carrera electoral entre Donald Trump y Hillary Clinton, la mentira distribuida masivamente por internet se ha convertido no sólo en una normalidad sino también en una nueva arma, muy potente, de propaganda (Journell, 2017). Autores como Mcnair (2018) sitúan el fenómeno en el contexto del auge de los populismos y los nacionalismos y el desprestigio de las élites y los medios de comunicación.

Ante este panorama de crisis de confianza en los sistemas convencionales de información y comunicación, se puede plantear qué papel debería jugar el profesional de la información y la documentación. A nuestro entender se presenta un doble reto:

- cómo determinar el grado de confianza de una fuente de información;
- cómo tratar de revertir la situación en lo posible, es decir cómo combatir la viralización cual tsunami de una información falsa.

Como hemos comentado, hasta ahora siempre ha sido misión de los bibliotecarios formar a los usuarios en el uso de las fuentes de información. Éste vuelve a ser el mayor reto, ya que la mayoría de las personas de nuestro entorno se informan mediante alguna red social (WhatsApp, Twitter, Facebook, etc.) mucho antes que a través de los medios de comunicación tradicionales (Shearer; Gottfried, 2017).

En este trabajo presentaremos una visión general del fenómeno de las noticias falsas (definición, tipología, etc.) y una serie de propuestas que tienen que ver con los valores y capacidades de nuestra profesión para luchar contra un virus que cada vez se extiende más y contra el que el profesional de la información y documentación puede tener un papel determinante.

\section{La verdad y la posverdad, herramienta de opinión y de lucha ideológica}

Se ha empezado a hablar mucho de fake news (noticias falsas) después de la campaña electoral de las elecciones presidenciales de Estados Unidos de 2016 (Allcott; Gentzkow, 2017).

Medios de comunicación que airean noticias falsas y con muy baja credibilidad han existido siempre, pero con las actuales facilidades para crear y difundir contenidos ha aparecido una práctica relacionada con la parodia, la rumorología o directamente con intereses políticos, sociales o económicos, de la que sólo podemos constatar su aumento, aunque a estas alturas somos incapaces de predecir sus efectos (Burkhardt, 2017). 
Tabla 1. Etiquetas de clasificación de OpenSources.co, un portal de listas curadas de fuentes online. http://www.opensources.co

\begin{tabular}{|c|c|}
\hline Etiqueta & Descripción \\
\hline Fake news & Fuentes que fabrican información por completo, difunden contenido engañoso o distorsionan las noticias reales \\
\hline Sátira & Fuentes que usan humor, ironía, exageración, ridículo e información falsa para comentar sobre eventos actuales \\
\hline Sesgo extremo & $\begin{array}{l}\text { Fuentes que provienen de un punto de vista particular y pueden basarse en la propaganda, la información descontex- } \\
\text { tualizada y las opiniones distorsionadas como hechos }\end{array}$ \\
\hline Teoría de la conspiración & Fuentes que son conocidas promotoras de teorías de conspiración \\
\hline Rumor & Fuentes que difunden rumores, chismes, insinuaciones y afirmaciones no verificadas \\
\hline State news & Fuentes en estados represivos que operan bajo la aprobación del gobierno \\
\hline Pseudociencia & Fuentes que promueven pseudociencia, metafísica, falacias naturalistas y otras afirmaciones científicamente dudosas \\
\hline Hate news & Fuentes que promueven activamente el racismo, la misoginia, la homofobia y otras formas de discriminación \\
\hline Clickbait & $\begin{array}{l}\text { Fuentes que proporcionan contenido generalmente creíble, pero usan titulares exagerados, engañosos o cuestiona- } \\
\text { bles, descripciones de redes sociales y / o imágenes }\end{array}$ \\
\hline Proceda con precaución & Fuentes que pueden ser confiables pero cuyos contenidos requieren una verificación posterior \\
\hline Política & $\begin{array}{l}\text { Fuentes que proporcionan información generalmente verificable en apoyo de ciertos puntos de vista u orientaciones } \\
\text { políticas }\end{array}$ \\
\hline
\end{tabular}

El nuevo marco mental que generó la campaña de Trump, tal como menciona Journell (2017), se basó en vincular la idea de fake a la información que contradice la propia ideología. Lo que lo hace peligroso es que la verdad no parece lo más relevante, sino lo accesorio. Y la verdad como valor ideológico deviene no ya subjetivo en la lógica partidista, sino objetivable en función de la ideología. Se puede relacionar esta deriva con la que también es posible comprobar en los debates de campaña en las diversas elecciones, donde los datos (bajadas de presupuestos, gráficas inverosímiles) se convierten en dardos lanzados entre candidatos. Así por ejemplo podemos ver campañas electorales basadas en visualizaciones e infografías (Gutiérrez-Rubí, 2012).

A ello cabe añadir que en el mundo de la comunicación hace tiempo que se ha roto el ciclo de producción de noticias que permitía su verificación. El modelo de información continua de 24 horas y las redes sociales obligan a estar generando y difundiendo noticias de manera permanente, siendo un campo abonado para la noticia falsa. Ahora mismo el concepto de noticia falsa puede llegar a ser polisémico, desde una parodia que todo el mundo sabe que es mentira hasta la creada expresamente, pasando por noticias publicadas con datos falsos para las que los controles de calidad de un determinado medio de comunicación no han sido los correctos. Según Rochlin (2017) una noticia falsa se puede definir como un titular y una historia deliberadamente falsa publicada en una web que quiere parecer un sitio de noticias reales. Zimdars (2016) definió 11 tipos posibles de sitios web de baja confianza, que son los utilizados por el portal OpenSources.co, dentro de las cuales englobaba las fake news (tabla 1).

\section{Medios sociales, entre la inteligencia artificial y la curación manual de contenidos}

Es en los medios sociales donde el debate sobre las noticias falsas, la posverdad y la utilización de noticias y desinformación para la manipulación del estado de opinión de la sociedad ha sido más relevante. Evidentemente, una de las causas es la gran presencia que tienen en la sociedad; sólo hay que ver las cifras de usuarios (gráfico 1). Por otra parte, están teniendo una presencia mediática e incluso política, cada vez mayor. Por ejemplo, en enero de 2018 Facebook ha tenido contactos con el fiscal especial nombrado por Donald Trump para estudiar la posible implicación de redes rusas para la creación de falsas noticias y múltiples perfiles y bots, todo bajo la sospecha de la intención de cambiar tendencias y el intento de influir en la campaña electoral americana donde ganó finalmente Trump (Lapowsky, 2018). Se valora que serían alrededor de 3.000 anuncios en Facebook

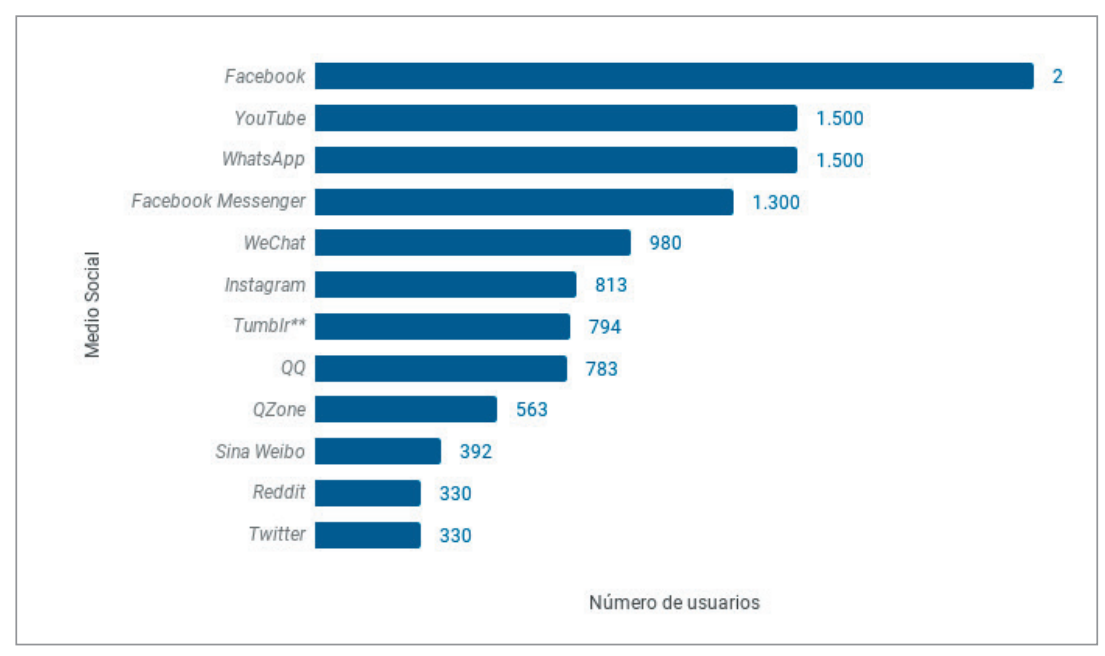

Gráfico 1. Medios sociales con más usuarios en abril de 2018 (en millones). Fuente: Statista https://www.statista.com/statistics/272014/global-social-networks-ranked-by-number-of-users 
e Instagram de cuentas falsas ligados a propaganda rusa, que habrían llegado, según los cálculos, a 126 millones de usuarios de Facebook (Bessi; Ferrara, 2016).

Para entender del todo el alcance de este hecho hay que considerar que Facebook, además de medio social, se convierte en sí mismo en un medio de comunicación donde los contenidos son aquello a difundir. El medio social se convierte en el lugar de referencia donde enterarse de lo que está pasando, por proximidad, por recomendación de otros usuarios o por cantidad de contenidos colgados. Así se posicionó en hechos como la primavera árabe, junto con Twitter (Bruns; Highfield; Burgess, 2013).

\section{Algunos autores sitúan el fenómeno de las noticias falsas en el contexto los populis- mos y los nacionalismos, y el desprestigio de las élites y los medios de comunicación}

El motivo por el que parte de la discusión y la veracidad afectan en mayor grado a los medios sociales se puede entender si tenemos en cuenta el gráfico 2. Autores del Pew Research Center, en un estudio en Estados Unidos en 2017, comprobaron que un porcentaje muy relevante de la sociedad empleaba Facebook para informarse de lo que estaba sucediendo (Shearer; Gottfried, 2017). Mientras el 66\% de los encuestados usa Facebook, el $45 \%$ lo hace para estar informado. No era la curiosidad de saber qué hacen los amigos, ni ver las fotografías de familiares y conocidos. Facebook se convertía en el medio.

La Comisión Europea (2018a) publicó una encuesta a nivel europeo sobre las fake news y la desinformación online. Los resultados destacan cómo dos terceras partes de los encuestados dijeron que al menos una vez a la semana se encuentran con fake news, y que existe una mayoría que cree que es un problema en su país para la propia democracia en general. Resulta también relevante que aunque la población tiene un nivel de confianza elevado en su habilidad para identificar noticias falsas, sólo un $15 \%$ se sienten muy seguros al respecto (Unión Europea, 2018). Dichos resultados son generales en todos los países y los distintos grupos sociodemográficos.

Tal como describen Alcott y Gentzkow (2017) en relación con las noticias falsas y los medios sociales, hay estudios (Silverman, 2016) que describen por ejemplo que la historia más popular calificada como fake new fue ampliamente más compartida en Facebook, que las noticias veraces más difundidas. También concluyeron que mucha gente que consume noticias falsas las cree e incluso, en la correlación entre Donald Trump y Hillary Clinton, la mayoría de las noticias falsas parecían favorecer más a Trump.
En este mismo sentido, un estudio publicado recientemente concluye que el contenido falso fue difundido más lejos, más rápido y más ampliamente que los contenidos ciertos con que lo compararon, siendo más importante el efecto cuando se trataba de noticias políticas sobre terrorismo, desastres naturales, ciencia o leyendas urbanas (Vosougui; Roy; Aral, 2018). De nuevo se concluyó que las noticias falsas más recientes eran difundidas más rápidamente. Éste y otros estudios pueden dar indicaciones de como reconducir los flujos de noticias falsas.

Considerando lo dicho, los medios sociales en general, pero sobre todo Facebook y Twitter, se han dado cuenta de que la falta de fiabilidad en los contenidos que difunden es una amenaza a su propia existencia. Si los medios sociales se convierten en sólo un altavoz de proximidad y rumores no contrastados, pierden parte de su esencia y sentido, y también su interés comercial. A continuación, se describen algunas de las propuestas e iniciativas que están considerando.

Facebook comenzó en octubre un experimento para bifurcar los contenidos de medios profesionales y los contenidos creados por los propios usuarios. Así, en Sri Lanka, Bolivia, Guatemala, Camboya, Serbia y Eslovaquia (el 1\% de la población mundial) hizo que en el news feed de los contactos sólo aparecieran contenidos creados por usuarios y anuncios, excluyendo los contenidos profesionales. Recientemente (marzo 2018) han decidido no seguir con el experimento al constatar que la experiencia no era la misma y había múltiples críticas por parte de los usuarios, así como de los periodistas y medios de aquellos países que veían muy reducido el tránsito hacia sus sitios web (Wong, 2018). En algunos entornos se han considerado estos experimentos como absolutamente orwellianos (downright Orwellian) (Hern, 2017).

Paralelamente, pero en la misma línea de encontrar una identidad y una experiencia clara (y fidelizadora) para el usuario, Mark Zuckerberg, creador de Facebook, explicó que uno de los retos de 2018 es que el tiempo que los usuarios

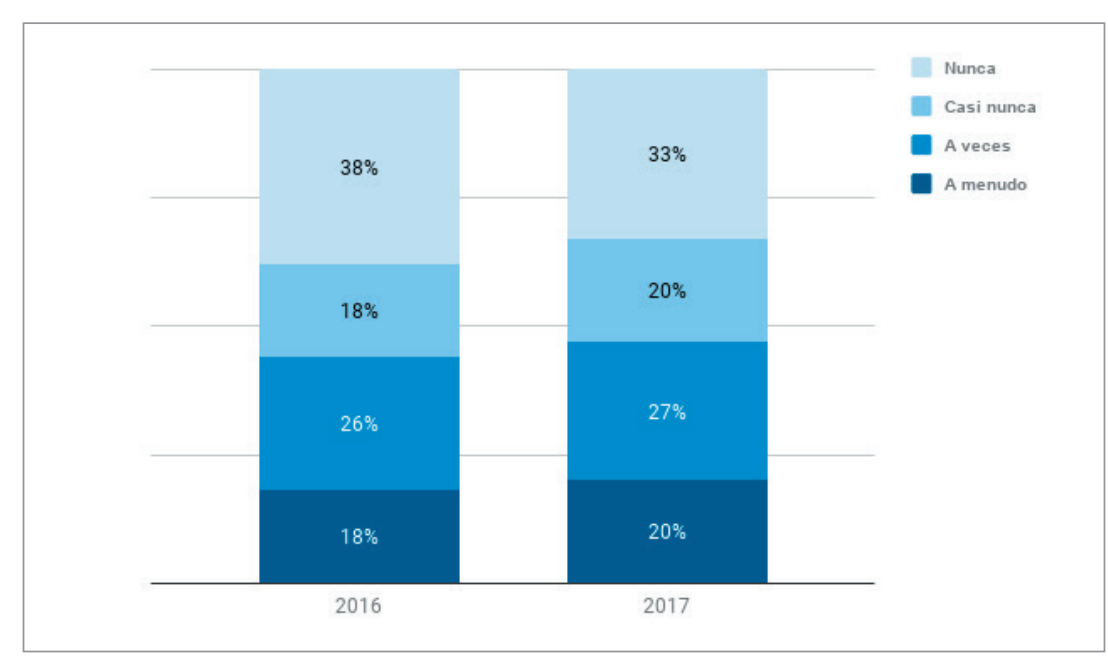

Gráfico 2. Porcentaje de adultos en Estados Unidos que siguen las noticias a través de los medios sociales (2016, 2017). Fuente: Pew Research Center

http://assets.pewresearch.org/wp-content/uploads/sites/13/2017/09/13163032/PJ_17.08.23_socialMediaUpdate_FINAL.pdf 
estén en el medio social sea bien empleado y valioso. https://www.facebook.com/zuck/posts/10104413015393571

En este sentido, se plantean incrementar los contenidos locales, de proximidad, lo que llama "promover la comunidad":

- depender menos de las grandes noticias y las olas informativas o eventos como una campaña electoral;

- más contenidos de familiares que de empresas y medios.

Es pronto para ver cómo reaccionarán los usuarios. La clave, como en toda curación y gestión de contenidos estará en un algoritmo que satisfaga tanto al usuario como a los inversores y las empresas de publicidad.

Lejos de asumir que es imposible luchar contra las noticias falsas, hay que reivindicar el papel de los profesionales de la información para formar usuarios críticos

Una de las claves de futuro de la relación entre los medios sociales y las noticias falsas vendrá relacionada con cómo se pueden identificar tecnológicamente o a nivel personal. Existen propuestas de data mining para la identificación de las noticias falsas (Shu et al., 2017), así como propuestas para crear una clasificación de los medios de comunicación, de las fuentes, en función de su fiabilidad (similar a la existente por los contenidos sensibles por sexo o violencia) que permita superar la dificultad de emplear herramientas de fact checking, que a menudo llegan muy tarde, cuando ya se ha difundido el contenido y por tanto mucha parte del daño ya está hecho (Kim; Moravec; Dennis, 2017).

Finalmente, otra de las preocupaciones de los medios sociales es la llamada lucha contra los bots, los robots y los perfiles falsos que difunden información. En este caso, no sólo estamos hablando de los contenidos que comparten, sino también de la modificación de la influencia que los usuarios pueden percibir en la Red. Una noticia compartida por un millón de usuarios, ¿es más cierta que una compartida por 100 usuarios? Evidentemente no, pero eso no impide que se puede crear una falsa sensación de autenticidad para usuarios que desconocen las fuentes o las formas de evaluarlas. Shao et al. (2017) han estudiado el fenómeno y han concluido que los social bots tienen un papel clave en la difusión de desinformación.

Facebook, en un estudio propio (Weedon; Nuland; Stamos, 2017), concluye que no serían los bots los principales contribuyentes a la difusión de noticias falsas y desinformación sino los propios usuarios. En este sentido, habría que pensar en un tipo de formación, una nueva alfabetización ligada a los usuarios para poder detectar las noticias falsas. También habría que estudiar si la propia radicalización de la opinión pública en determinados momentos (campaña electoral de Estados Unidos en 2016, referéndum del Brexit, referéndum catalán del 1 de octubre de 2017) generan un estado de tensión emocional que favorece la difusión de contenidos falsos o no suficientemente contrastados, o incluso tergiversados.
También Twitter como medio relevante para la creación de opinión pública está haciendo esfuerzos para ayudar a filtrar la aguja de la verdad en un pajar de fake news. Se han hecho estudios del uso de bots en varias campañas electorales, donde se calcula que hasta un $20 \%$ del tráfico podría provenir de bots sospechosos (Bessi; Ferrara, 2016). Algunos de los cambios que propone Twitter para evitar el mal uso de los bots son:

- no permitir colgar contenido idéntico o sustancialmente similar a múltiples cuentas;

- no permitir acciones como likes, retweets o follows desde múltiples cuentas (Roth, 2018).

Habrá que ver cómo afectan estas decisiones a los usos habituales de los bots y de programas como TweetDeck, así como al trabajo de gestión de comunidades por parte de profesionales de la información y la comunicación.

Lomas (2018) afirma que las noticias falsas representan una crisis existencial de los medios sociales. A la hora de buscar soluciones hay que tener en cuenta que el poder político ha estado en el punto de mira de los medios de comunicación y considerar también que las dos principales redes sociales, consideradas a veces como el quinto poder, a pesar de ser globales residen en un estado donde su presidente continúa señalando medios de comunicación cuya línea editorial no le gusta. Y eso, desgraciadamente puede tener consecuencias a nivel global.

Se vislumbra una doble estrategia por parte de los medios sociales. Ésta se basaría en la utilización conjunta de los algoritmos y la inteligencia artificial para filtrar automáticamente contenidos con el estudio de los contenidos reportados por usuarios. A este segundo fin parece que podría dedicarse el futuro centro a instalar por parte de Facebook en Barcelona.

\section{Alguna de las acciones que las bibliote- cas pueden llevar a cabo sería por ejem- plo difundir los servicios de verificación de datos (fact checks)}

Por su parte, Google también ha centrado sus esfuerzos en la mejora de su capacidad para filtrar contenidos considerados falsos mediante su proyecto Google News Initiative, que pretende trabajar en relación con los medios de comunicación ya existentes, al valorar que son los principales interesados en diferenciarse de emisores de fake news (Martens et al., 2018).

Además de los esfuerzos en el campo de las redes sociales, es destacable el trabajo llevado a cabo por las instituciones europeas sobre la necesidad de una visión consensuada del análisis del fenómeno y los intentos de regulación. Así, en el informe de la Comisión Europea (2018b) Ilamado La lucha contra la desinformación en línea: un enfoque europeo se considera que

"la exposición de los ciudadanos a una desinformación a gran escala, que incluye información engañosa o completamente falsa, representa un gran reto para Europa" (Comisión Europea, 2018b). 
Asimismo, un grupo de alto nivel ha estado trabajando en este asunto, con presencia española del medio Maldito bulo. En dicho grupo de expertos destacan los profesionales de la Comunicación, pero se echan en falta colectivos del mundo bibliotecario que podrían enriquecer la visión y las propuestas de solución.

https://ec.europa.eu/digital-single-market/en/news/expertsappointed-high-level-group-fake-news-and-online-disinformation

Por otra parte, varios estados miembros de la UE han estado trabajando en regulaciones propias. Hay que destacar los debates en Francia y Alemania.

https://www.politico.eu/article/french-parliament-passeslaw-against-fake-news

https://www.bbc.com/news/technology-42510868

En el caso francés, la propuesta de ley permite que en período electoral se pueda pedir que sean eliminados determinados contenidos. Por su parte, Alemania ha legislado en la línea de imponer fuertes multas a los servicios de contenidos que sean responsables de la difusión de contenidos falsos.

Finalmente hay que destacar algunas de las aportaciones que investigadores como Tambini (2017) han llevado a cabo: propone para los legisladores que las políticas deben incentivar la alfabetización, y la autorregulación por parte de las plataformas, a la vez que defienden que las soluciones no deberían pasar por aumentar las responsabilidades sobre los medios de comunicación.

¿Por qué no un laboratorio ciudadano de verificación de noticias falsas de proximidad dentro de una biblioteca?

\section{Neutralidad bibliotecaria, verdad y fake news}

No trataremos aquí de definir qué es la verdad y que la falsedad, basta con aceptar que, a veces, dos usuarios de la misma biblioteca o sistema bibliotecario pueden no coincidir en sus respectivas apreciaciones sobre qué es o no es en función de sus propios criterios. En un artículo reciente se cuestionaba que las bibliotecas públicas españolas tuvieran libros sobre lo que los autores llamaban falsa ciencia y comparaban la disponibilidad de títulos de homeopatía con los documentos sobre quimioterapia (Cortiñas-Rovira; Darriba-Zaragoza, 2018). Una de las opiniones, discutibles, de estos autores es que

“...la mera posibilidad de poner a disposición de ciudadanos no formados en ciencia, información sobre aspectos de medicina no verificados, supone una amenaza para la salud de la ciudadanía y pone en entredicho la función de cohesión social que Solimine (2012) otorga a las bibliotecas públicas".

https://twitter.com/pepvivesBIB/status/969331832251666433

En la misma línea, otros usuarios planteaban a través de Twitter la idoneidad de disponer en las bibliotecas públicas títulos como Los niños con los niños, las niñas con las niñas o El libro de las niñas. Como no podía ser de otra forma, tampoco falta quienes se preguntan por qué en las bibliotecas públicas hay diarios que algunas personas consideran poco objetivos y piden su retirada. Pensamos que cuanto más importante sea el papel de las bibliotecas en la sociedad y su presencia, más estaremos sometidos a este tipo de cuestiones y por tanto es necesario que los profesionales tengamos claro cómo proceder. De hecho, se podría pensar que el tema de las noticias falsas es sólo la punta del iceberg que lentamente se abre ante la sociedad.

Hay un aspecto que diferencia claramente el acceso a la información a través de un buscador, del acceso a través de una biblioteca: la selección. Una biblioteca concreta, por muchos recursos que tenga nunca podrá poner a disposición de sus usuarios todos los aspectos relacionados con un determinado tema (Anderson, 2017). A partir de ahí, ¿qué tenemos que hacer, mantener una estricta neutralidad y dar por hecho que el usuario será capaz por sí solo de analizar la información de una manera crítica, o bien deberíamos disponer de filtros en las colecciones? Por otra parte, ¿no es la neutralidad por sí misma ya una especie de posicionamiento?

\section{¿Debemos tener entre nuestras coleccio- nes documentos que son absolutamente falsos y confiar en la capacidad del usuario y en nuestros programas de alfabetización en información o por el contrario debemos tener sólo información de calidad, aunque no sea la más usada?}

Anderson (2017) propone cuatro argumentos interesantes para situar la discusión:

- existe la verdad objetiva;

- los seres humanos están capacitados para reconocerla de manera fiable;

- hay que esperar que las bibliotecas ayuden a sus usuarios a distinguir entre la verdad objetiva y la falsedad y que piensen críticamente por ellos mismos (antes que conducir a los usuarios hacia una posición social o política determinada);

- en las bibliotecas no se intenta evitar que los usuarios encuentren falsedades, sino que se hace lo posible para ayudarles a leer críticamente y llegar a conclusiones válidas y bien informadas sobre lo que es verdadero y bueno y lo que es falso y malo.

Si aceptamos estos argumentos, una pregunta obvia que uno se podría plantear desde la perspectiva del bibliotecario-documentalista es si hay que hacer algo en relación con las noticias falsas y por qué. Sabemos que una de las máximas éticas de nuestra profesión es la neutralidad entendida como que

"Ios bibliotecarios y otros trabajadores de la información están estrictamente comprometidos con la neutralidad y con una postura imparcial en relación a la colección, el acceso y el servicio. La neutralidad tiene como resultado el mayor equilibrio posible en la colección y en el acceso a la información" (IFLA, 2012).

Al mismo tiempo los bibliotecarios-documentalistas "apo- 
yan a los usuarios en su búsqueda de información" y "ofrecen servicios para incrementar las habilidades de lectura", especialmente

"promueven la alfabetización informacional incluyendo la capacidad para identificar, localizar, evaluar, organizar y crear, utilizar y comunicar información" (IFLA, 2012).

Uno de los objetivos de la alfabetización informacional es precisamente crear usuarios críticos en el uso de la información y, por supuesto, que sean capaces de distinguir fuentes de información fiables de las que no lo son. Esto forma parte de este aprendizaje que los profesionales de la información apoyamos. Según Becker (2016), las noticias falsas ya aparecían como tendencia en 2004 enmarcadas en programas televisivos satíricos, los cuales se jactaban de ser líderes en este tipo de "noticias". La cuestión se circunscribía a este tipo de medios. En 2018, sin embargo, se ha empezado a hablar de epidemia desinformativa.

Para los profesionales de la información ésta no es una nueva guerra, sino que llevamos más de 30 años situando este problema en algunos de nuestros ejes profesionales (Becker, 2016). En 1989, la Association of College \& Research Libraries (ACRL) determinaba que

"ciudadanía en democracia moderna es más que saber acceder a información vital. También incluye la capacidad de reconocer propaganda, distorsión y otros usos indebidos y abusos de la información" (ACRL, 1989),

y fijaba claramente lo que ha sido uno de los objetivos hasta ahora de las bibliotecas:

"Las bibliotecas públicas no sólo proporcionan acceso a la información, sino que también siguen siendo cruciales para proveer a las personas los conocimientos necesarios para hacer un uso adecuado de los recursos existentes. Siguen siendo una de las pocas salvaguardas contra el control de la información por una minoría" (ACRL, 1989).

La clave es reforzar los programas de alfabetización informacional y adaptarlos a las necesidades actuales, no se trata tanto de "contraatacar" las noticias falsas con otras noticias y entrar en discusión con los usuarios, sino de concienciar y formar lectores más críticos y más consumidores de información de fuentes diversas (Finley; McGowan; Kluever, 2017). Como ejemplo de este camino podemos mencionar la gestión ejemplar a través de las redes de la información relativa a los atentados de Barcelona y Cambrils de 2017. El mensaje era muy claro y fácil de hacer llegar: hacer caso sólo de las informaciones que llegan a través de los canales oficiales de emergencias (Catà, 2017).

\section{Proyectos e iniciativas en el mundo de la información y la documentación}

En el sector de la información y la documentación se han generado diversos proyectos e iniciativas en los últimos tiempos. En este sentido, la International Federation of Library Associations and Institutions (IFLA) ha creado una infografía (gráfico 3): Cómo detectar noticias falsas, que se ha convertido en viral.

Como apunta López-Borrull (2017), puede ser de gran utili-
HOW TO SPOT FAKE NEWS

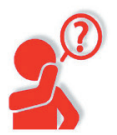

CONSIDER THE SOURCE

Click away from the story to investigate the site, its mission and its contact info.

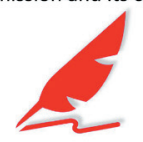

CHECK THE AUTHOR

Do a quick search on the author. Are they credible? Are they real?

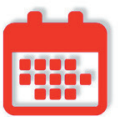

CHECK THE DATE

Reposting old news stories doesn't mean they're relevant to current events.

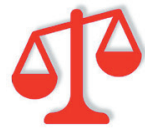

CHECK YOUR BIASES

Consider if your own beliefs could affect your judgement.

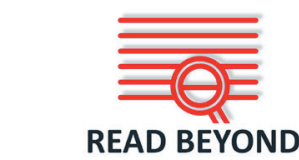
to get clicks. What's the whole story?

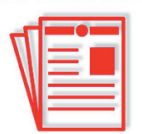

SUPPORTING SOURCES?

Click on those links. Determine if the info given actually supports the story.

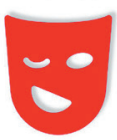

IS IT A JOKE?

If it is too outlandish, it might be satire.

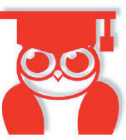

ASK THE EXPERTS

Ask a librarian, or consult a fact-checking site.
Headlines can be outrageous in an effort Research the site and author to be sure.

DFLa

Gráfico 3. Infografía creada por la IFLA para luchar contra las fake news. https://www.ifla.org/publications/node/11174

dad sobre todo porque tiene esa viralidad para llegar donde se están difundiendo informaciones poco precisas o falsas. Según definición de la propia IFLA,

“...una simple pero efectiva herramienta que ofrece una alternativa, basada en la convicción de que la educación es la mejor forma de que los usuarios adquieran confianza y de que los gobiernos disculpen una censura innecesaria".

La infografía ha tenido un gran impacto a nivel mundial, y ha sido noticia en la CNN Internacional, entre otros medios.

Por otra parte, el movimiento bibliotecario ha sido activo como reacción al fenómeno: la biblioteca del Parlamento de Finlandia la presentó en una reunión parlamentaria; también en Suecia, Alemania, Vietnam o Malasia. Asimismo, en Cataluña, se ha hecho difusión y se han colgado carteles y posters, se han organizado debates y se han generado artículos y publicaciones. Como ejemplo, destacar la conferencia sobre noticias falsas en el encuentro de la XBM (Red de Bibliotecas Municipales de la Diputación de Barcelona) celebrada a mediados de febrero de 2018. Y las XXIV Jornadas de Comunicación Blanquerna (marzo 2018, URL) estuvieron centradas en la posverdad.

Además de esta iniciativa, la IFLA ha lanzado una campaña que anima a sus miembros a participar en debates sobre los medios de comunicación y sobre la alfabetización informacional en todo el mundo para llamar la atención sobre el papel fundamental que esta institución cree que las biblio- 
tecas pueden y deben realizar. A finales de febrero de 2018, la misma IFLA con la Unión Europea organizó un coloquio en Bruselas para debatir específicamente el problema de la desinformación por internet. Una de las conclusiones principales fue que hay que ir hacia políticas de formación en habilidades digitales y no tanto a restricción o prohibición de acceso. Aquí las bibliotecas y los bibliotecarios tienen un papel capital. Las noticias falsas son tema de debate en la mayoría de jornadas y reuniones internacionales de las secciones y organizaciones nacionales de la IFLA.

La Unesco, por su parte, ha reiterado su compromiso de trabajo y esfuerzo con la Alfabetización Mediática e Informacional / Media and Information Literacy (AMI/MIL) como uno de los requisitos más importantes para fomentar el acceso equitativo a la información y al conocimiento, y para promover los medios de comunicación y los sistemas de información libres, independientes y pluralistas. La AMI contempla todos los tipos de medios de comunicación y otros proveedores de información como bibliotecas, archivos, museos e internet, independientemente de las tecnologías utilizadas.

La Cilip (The Library and Information Association) modificó en abril de 2018 su definición de 2004 sobre la alfabetización informacional (alfin) para adaptarla al fenómeno de la información falsa:

"La alfabetización informacional es la habilidad de pensar críticamente y emitir juicios equilibrados sobre cualquier información que encontremos y utilicemos. Es empoderarnos como ciudadanos para alcanzar y expresar puntos de vista informados y comprometernos plenamente con la sociedad" (Cilip, 2018).

En España, el Consejo de Cooperación Bibliotecaria (CCB, 2016), organismo dependiente del Ministerio de Cultura, y en concreto, el Grupo de Trabajo sobre Alfabetización Mediática e Informacional ha elaborado el informe Integración de las competencias alfin/AMI en el sistema educativo: referencias, contexto y propuestas, donde se recogen 10 medidas urgentes para la integración efectiva de dichas competencias. Van dirigidas a profesores, directores de centros educativos, servicios de formación, inspección educativa y bibliotecarios escolares para que vean la importancia de estas recomendaciones y actúen en consecuencia.

El mismo organismo, en su II Plan estratégico, ponía el foco de atención e importancia en el papel de la biblioteca escolar en la adquisición de las competencias AMI. Ello coincide con la visión recogida por Tiffany (2016), que indica que los bibliotecarios escolares tienen un papel importante. En España, colectivos de profesionales trabajan desde hace tiempo en la formación para la adquisición de las competencias informacionales, digitales o mediáticas (como últimamente son conocidas, media literacy):

- Comunidad Baratz: que publica un blog muy seguido y artículos sobre la temática;

- Bibliomèdia: grupo de la Federación de Movimientos de Renovación Pedagógica de Cataluña, también trabaja en esta misma línea.

En todo caso, como comenta Alsius (2017), la educación mediática aparece como una condición no suficiente pero sí absolutamente necesaria para cambiar el estado de las cosas. La adopción en masa de las tecnologías relacionadas con internet ha cambiado la manera de crear y consumir noticias. Según Chen, Conroy y Rubin (2015) el entorno actual de noticias online es lo que incentiva la velocidad; y el espectáculo en los contenidos generados por el usuario y las noticias tradicionales se ha vuelto cada vez más borroso. Estos autores proponen la creación de un programa informático de detección automática de noticias falsas como complemento a las políticas de formación mediáticas. Argumentan una doble acción para combatir el problema:

- compromiso público proactivo de educadores, bibliotecarios y profesionales de los medios de comunicación para promover prácticas de alfabetización mediática;

- desarrollo de aplicaciones y tecnologías automáticas que ayuden a velar, verificar y comprobar hechos y ayudar a los lectores filtrando y marcando información dudosa. En este último punto, la propuesta coincide con los anuncios que Google, Facebook y Twitter están llevando a cabo y que se han comentado anteriormente.

Se tendrá que trabajar tal vez más activamente que hasta ahora en la alfabetización mediática desde los primeros estadios del sistema educativo. Sobre todo teniendo en cuenta los resultados de varios estudios que determinan la infrautilización de los recursos y fuentes de información por parte de los estudiantes universitarios (Cid-Leal; Perpinyà-Morera, 2015). Un estudio de estas autoras centrado en el grado de Traducción e Interpretación, pero perfectamente extrapolable a todos los estudiantes universitarios, concluye que

"las bibliotecas universitarias no son el referente principal" y que la "Red se ha convertido en el recurso principal para buscar información académica".

Además, Cid-Leal y Perpinyà-Morera señalan también que

"los estudiantes prefieren recursos y fuentes de información relacionados con la facilidad de obtención por encima de los criterios de calidad".

En paralelo, un estudio que analizaba los contenidos de 517 tutoriales de programas de alfabetización informacional de 52 instituciones norteamericanas constataba que sólo el 1,4\% incluía el estudio de las noticias falsas (Saunders, 2017). La autora del estudio indicaba la necesidad de prestar atención a esta nueva casuística ya que actualmente el problema no es tanto encontrar información sino cómo evaluarla.

\section{Profesionales de la información en relación con las fake news. Conclusiones y propuestas}

Los profesionales de la información han vivido diferentes tsunamis que históricamente han impedido o dificultado uno de sus objetivos básicos: poner a disposición de los usuarios la información que necesitan en cada momento. La censura fue seguramente el más importante de estos impedimentos ya que el problema no era no poder disponer de una cantidad importante de información que gracias a la invención de la imprenta se podía reproducir fácilmente, sino los impedimentos legales, morales y religiosos para hacerlo. 
Una vez superada la censura, aunque nunca eliminada del todo, los profesionales de la información tuvieron que enfrentarse a la ingente cantidad de información disponible.

Aún no resuelto este problema, aparece una nueva variable. Algunas de estas informaciones pueden ser falsas o sesgadas y en muchos casos no por error, sino de manera intencionada. Se podría pensar que tal vez se abra una nueva oportunidad de situar al profesional de la información allí donde siempre ha estado, sumergido en el mundo de la información, eligiendo, organizando, conservando y ayudando a los usuarios a encontrar lo que necesitan. Farmer (2017) destaca que las habilidades adquiridas por los bibliotecarios formadores en alfabetización de medios los hacen especialmente adecuados para acompañar a los estudiantes en este proceso.

Mediante la formación y la organización de actividades de sensibilización es como se sitúa el debate donde corresponde, no con la censura de las colecciones

Las noticias falsas han venido para quedarse y habrá que desplegar y reforzar una serie de estrategias profesionales de las que los profesionales son expertos, sobre todo las relacionadas con la formación de los usuarios en el uso de la información y por extensión en la validación de sus fuentes. En paralelo será necesario seguir ampliando el espectro ideológico de las colecciones. Las noticias falsas no se combaten con la censura sino precisamente con más noticias y más formación en fuentes de información. Los profesionales de la información deben ser conscientes de que mantener un espectro amplio en las políticas de adquisición hace que se acaben incorporando a las colecciones (especialmente en las bibliotecas públicas) documentos que no siempre reúnen los requisitos formales que se pediría a una fuente académica o de referencia que, por otro lado tiene la obligación de mostrar la diversidad de opiniones y sensibilidades de la sociedad a la que sirve. Este no es un problema nuevo; existen libros y otros documentos en las colecciones que no garantizan su objetividad, pero se ha aprendido a empoderar a los usuarios en este sentido y a ofrecerles la posibilidad de elegir (Finley; McGowan; Kluever, 2017). Mediante la formación y la organización de actividades de sensibilización es como se sitúa el debate donde corresponde, no con la censura de las colecciones.

Los pasos a seguir desde una visión profesional pueden ser diversos. Según Johnson (2017), las bibliotecas tienen una de las cosas más preciadas en el mundo, una marca respetada y en la que los usuarios confían. ¿Deben existir entre las colecciones documentos que son absolutamente falsos y confiar en la capacidad del usuario y en nuestros programas de alfabetización informacional o por el contrario se debe tener sólo información de calidad, aunque no sea la más usada? El debate sigue y seguirá abierto.

Si se asume el papel de los profesionales de la información y la documentación como expertos en lo que se ha dado en llamar "razonamiento cívico online" (Wineburg et al., 2016) son muchas las estrategias que se pueden incorporar en los actuales planes de alfabetización informacional y alfabetización de medios.

Farmer (2017) propone algunas herramientas para trabajar dentro del ámbito de la enseñanza pero que fácilmente son extrapolables a otros colectivos de usuarios:

- criterios para evaluar noticias y otras fuentes de información;

- utilizar sitios web de comprobación de hechos (fact checkers);

- leer fuentes acreditadas;

- buscar diferentes perspectivas;

- practicar la alfabetización de medios;

- reducir la compartición de noticias dudosas;

- apoyar y comprometerse con el periodismo de alta calidad.

Otras acciones que creemos necesarias para ir incorporando a las agendas de bibliotecas podrían ser:

- disponer de materiales de formación online con ejemplos de noticias falsas y fuentes de información fiables;

- difundir los servicios de verificación de datos (fact checkers) y crear vías de colaboración con los ciudadanos (¿por qué no un laboratorio ciudadano de verificación de noticias falsas de proximidad dentro de una biblioteca?);

- organizar talleres prácticos sobre detección de noticias falsas;

- reforzar las colecciones, especialmente las digitales, con suscripciones a medios de comunicación de alta calidad, teniendo en cuenta que muchos de los medios digitales que más noticias falsas difunden son de acceso libre mientras que en periódicos de gran prestigio sólo se puede acceder mediante una suscripción y por tanto no son accesibles para la mayoría;

- establecer sinergias con profesionales de la comunicación para crear proyectos en común;

- reforzar el conocimiento de los códigos éticos profesionales por parte de nuestros profesionales para que les ayuden a definir las políticas de adquisiciones.

Las bibliotecas tienen una de las cosas más preciadas en el mundo: una marca respetada y la que los usuarios confían

Como afirma Rochlin (2017) estamos en una guerra de información. Lejos de asumir que es imposible luchar contra las noticias falsas, hay que reivindicar el papel de los profesionales de la información como generales en esta batalla a través de lo que siempre hemos hecho: formar usuarios críticos. Es nuestra gran arma. De verdad, esta sí.

\section{Referencias}

Allcott, Hunt; Gentzkow, Matthew (2017). "Social media and fake news in the 2016 election". Journal of economic perspectives, v. 31, n. 2, pp. 211-236. https://doi.org/10.1257/jep.31.2.211

Alsius, Salvador (2017). "De la postveritat a la postètica". 
BiD: textos universitaris de biblioteconomia i documentació, n. 39.

https://doi.org/10.1344/BiD2017.39.4

Anderson, Rick (2017). "Fake news and alternative facts: Five challenges for academic libraries". Insights, v. 30, n. 2, pp. 4-9.

https://doi.org/10.1629/uksg.356

ACRL (1989). Presidential Committee on Information Literacy: Final report. Association of College \& Research Libraries. http://www.ala.org/acrl/publications/whitepapers/presidential

Becker, Bernd W. (2016). "The librarian's information war". Behavioral \& social sciences librarian, v. 35 , n. 4, pp. 188 191.

https://doi.org/10.1080/01639269.2016.1284525

Bessi, Alessandro; Ferrara, Emilio (2016). "Social bots distort the 2016 U.S. Presidential election online discussion". First Monday, v. 21, n. 11.

http://firstmonday.org/article/view/7090/5653

Burkhardt, Joanna M. (2017). "Combatting fake news in the digital age". Library technology reports, v. 53, n. 8.

https://www.alastore.ala.org/content/combating-fakenews-digital-age

https://journals.ala.org/index.php/ltr/article/download/6497/8631

Bruns, Axel; Highfield, Tim; Burgess, Jean (2013). "The Arab Spring and social media audiences: English and Arabic Twitter users and their networks". American behavioral scientist, v. 57, n. 7 , pp. $871-898$.

https://doi.org/10.1177/0002764213479374

Casero-Ripollés, Andreu; López-Meri, Amparo (2015). “Redes sociales, periodismo de datos y democracia monitorizada". En: Campos-Freire, Francisco; Rúas-Araújo, José (eds.). Las redes sociales digitales en el ecosistema mediático. La Laguna: Sociedad Latina de Comunicación Social, pp. 96-113. https://doi.org/10.4185/cac92

Catà, Josep (2017). "Les claus de l'èxit de la comunicació dels Mossos durant els atemptats". El país, 23 agosto. https://goo.gl/NiyJBT

Chen, Yimin; Conroy, Niali J.; Rubin, Victoria L. (2015). "News in an online world: The need for an 'automatic crap detector'". In: Procs of the Association for Information Science and Technology, v. 52, n. 1, pp. 1-4.

https://doi.org/10.1002/pra2.2015.145052010081

Cid-Leal, Pilar; Perpinyà-Morera, Remei (2015). “Competència informacional en traducció: anàlisi dels hàbits dels estudiants universitaris en la consulta i l'ús de fonts d'informació". BiD: textos universitaris de biblioteconomia i documentació, n. 34.

https://doi.org/10.1344/BiD2015.34.10

Cilip (2018). Definition of information literacy. Cilip. The Library and Information Association; Information Literacy Group. https://infolit.org.uk/ILdefinitionCILIP2018.pdf

Comisión Europea (2018a). Fake news and disinformation online. Flash Eurobarometer 464.

http://ec.europa.eu/commfrontoffice/publicopinion/index. cfm/ResultDoc/download/DocumentKy/82797
Comisión Europea (2018b). La lucha contra la desinformación en línea: un enfoque europeo. $\operatorname{COM(2018)~} 236$ final. https://eur-lex.europa.eu/legal-content/ES/TXT/ PDF/?uri=CELEX:52018DC0236\&from=en

CCB (2016). Integración de las competencias alfin/AMI en el sistema educativo: referencias, contexto y propuestas. Madrid: Ministerio de Educación, Cultura y Deporte. Consejo de Cooperación Bibliotecaria.

http://www.ccbiblio.es/wp-content/uploads/Integracion_ competencias_ALFIN-AMI_-sistema_educativo.pdf

Cortiñas-Rovira, Sergi; Darriba-Zaragoza, Marc (2018). "Análisis de la presencia de pseudociencia en los catálogos de las bibliotecas públicas españolas". Revista española de documentación científica, v. 41, n. 1, e197. https://doi.org/10.3989/redc.2018.1.1474

Farmer, Lesley S. J. (2017). "Don't get faked out by the news: Preparing informed citizens". CSLA journal, v. 41, n. 1, pp. 12-13.

http://csla.net/wp-content/uploads/2012/09/CSLA_ Journal_41-1_-2017_v2.pdf

Finley, Wayney; McGowan, Beth; Kluever, Joanna (2017). "Fake news: An opportunity for real librarianship". ILA reporter, v. XXXV, n. 3, May 17.

https://www.ila.org/publications/ila-reporter/article/64/ fake-news-an-opportunity-for-real-librarianship

Gutiérrez-Rubí, Antoni (2012). “Infografías, visualizaciones y política”. El país. Blogs política. Micropolítica, 9 abril.

http://blogs.elpais.com/micropolitica/2012/04/infografiasvisualizaciones-y-politica.html

Hern, Alex (2017). "'Downright Orwellian': Journalists decry Facebook experiment's impact on democracy". The guardian, October $25^{\text {th }}$.

https://www.theguardian.com/technology/2017/oct/25/ facebook-orwellian-journalists-democracy-guatemala-slovakia

IFLA (2012). IFLA code of ethics for librarians and other information workers.

https://www.ifla.org/faife/professional-codes-of-ethics-forlibrarians

Johnson, Ben (2017). "Information literacy is dead: The role of libraries in a post-truth world". Computers in libraries, v. 37, n. 2.

http://www.infotoday.com/cilmag/mar17/Johnson-Information-Literacy-Is-Dead--The-Role-of-Libraries-in-a-PostTruth-World.shtml

Journell, Wayne (2017). "Fake news, alternative facts, and Trump: Teaching social studies in a post-truth era". Social studies journal, v. 37, n. 1, pp. 8-21.

http://www.uncg.edu/ awjourne/Journel/2017ssj.pdf

Kim, Antino; Moravec, Patricia; Dennis, Aalan R. (2017). "Behind the stars: The effects of news source ratings on fake news in social media". Kelley school of business research. Paper n. 18-3.

https://doi.org/10.2139/ssrn.3090355

Lapowsky, Issie (2018). "Mueller's team has interviewed Facebook staff as part of Russia probe". Wired, January $26^{\text {th }}$. 
https://www.wired.com/story/robert-mueller-russiainvestigation-facebook

Lomas, Natasha (2018). "Fake news is an existential crisis for social media". Techcrunch.

https://techcrunch.com/2018/02/18/fake-news-is-an-existential-crisis-for-social-media

López-Borrull, Alexandre (2017). “De posverdad, ¿que no tenemos nada que decir?". Comeln. Revista dels Estudis de Ciències de la Informació i la Comunicació, n. 64. http://comein.uoc.edu/divulgacio/comein/es/numero64/ articles/De-posverdad.html

McNair, Brian (2018). Fake news: Falsehood, fabrication and fantasy in journalism. London: Routledge. ISBN: 9781 138306790

Martens, Bertin; Aguiar, Luis; Gómez, Estrella; Mueller-Langer, Frank (2018). "The digital transformation of news media and the rise of disinformation and fake news". Digital economy working paper 2018-02. Joint Research Centre Technical Reports.

https://doi.org/10.2139/ssrn.3164170

Rochlin, Nick (2017). "Fake news: Belief in post-truth". Library hi tech, v. 35, n. 3, pp. 386-392.

https://doi.org/10.1108/LHT-03-2017-0062

Roth, Yoel (2018). "Automation and the use of multiple accounts". Twitter. Developer blog, February $21^{\text {st }}$.

https://blog.twitter.com/developer/en_us/topics/tips/2018/ automation-and-the-use-of-multiple-accounts.html

Saunders, Laura (2018). "Information literacy in practice: Content and delivery of library instruction tutorials". The journal of academic librarianship, v. 44, n. 2, pp. 269-278. https://doi.org/10.1016/j.acalib.2017.12.022

Shao, Chengcheng; Ciampaglia, Giovanni L.; Varol, Onur; Yang, Kaicheng; Flammini, Alessandro; Menczer, Filippo (2017). The spread of misinformation by social bots. https://arxiv.org/pdf/1707.07592.pdf

Shearer, Elisa; Gottfried, Jeffrey (2017). News use across social media platforms 2017. Pew Research Center.

https://goo.gl/qi6N9V
Shu, Kai; Sliva, Amy; Wang, Suhang; Tang, Jiliang; Liu, Huan (2017). "Fake news detection on social media: A data mining perspective". ACM Sigkdd explorations newsletter archive, v. 19, n. 1, pp. 22-36.

https://arxiv.org/abs/1708.01967

Silverman, Craig (2016). "This analysis shows how fake election news stories outperformed real news on Facebook". BuzzFeed news, November 16.

https://goo.gl/1Mn7th

Solimine, Giovanni (2012). "El conocimiento como bien común y el papel de las bibliotecas". Anales de documentación, v. 15, n. 1.

https://doi.org/10.6018/analesdoc.15.1.142761

Tambini, Damian (2017). Fake news: Public policy responses. Media policy brief 20. London: Media Policy Project, London School of Economics and Political Science.

https://goo.gl/6dvMtT

Tiffany, Kaitlyn (2016). "In the war on fake news, school librarians have a huge role to play". The verge, November $16^{\text {th }}$.

https://www.theverge.com/2016/11/16/13637294/schoollibraries-information-literacy-fake-news-election-2016

Vosougui, Soroush; Roy, Deb; Aral, Sinan (2018). "The spread of true and false news online" Science, v. 359, n. 6380, pp. 1146-1151.

https://doi.org/10.1126/science.aap9559

Weedon, Jen; Nuland, William; Stamos, Alex (2017). Information operations and Facebook. Facebook Security. https://fbnewsroomus.files.wordpress.com/2017/04/ facebook-and-information-operations-v1.pdf

Wineburg, Sam; McGrew, Sarah; Breakstone, Joel; Ortega, Teresa (2016). Evaluating information: The cornerstone of civic online reasoning.

http://purl.stanford.edu/fv751yt5934

Wong, Julia-Carrie (2018). "Facebook ending news feed experiment condemned as 'Orwellian'”. The guardian, March $1^{\text {st }}$. https://www.theguardian.com/technology/2018/mar/01/ facebook-news-feed-experiment-media-posts

\section{EP El profesional de la información http://www.elprofesionaldelainformacion.com/autores.html}

PRÓXIMOS TEMAS

\begin{tabular}{|c|l|l|r|}
\hline Número & Mes año & \multicolumn{1}{|c|}{ Tema } & Envío textos \\
\hline 28,1 & Ene 2019 & Multidisciplinar & 10 nov 2018 \\
\hline 28,2 & Mar 2019 & Información y comunicación biomédica & 10 nov 2018 \\
\hline 28,3 & May 2019 & Posverdad y desinformación & 10 ene 2019 \\
\hline 28,4 & Jul 2019 & Uso de información académica & 10 mar 2019 \\
\hline 28,5 & Sept 2019 & Comunicación organizacional & 10 abr 2019 \\
\hline 28,6 & Nov 2019 & Estructuras de poder en Comunicación & 10 jun 2019 \\
\hline
\end{tabular}


